Aus der Chirurgischen Universitätsklinik Kiel. (Vorstand: Geheimrat Prof. Dr. Ans chütz.)

\title{
Ein Beitrag zur Behandlung der verzögerten Knochenheilung und Pseudarthrose.
}

\author{
Von Prof. Dr. Max Kappis, Oberarzt der Klinik.
}

Unter Pseudarthrose versteht man allgemein den $\mathrm{Zu}$. stand, bei dem die knöcherne Heilung eines Knochenbruches ausgeblieben ist und keine Heilungsvorgänge mehr vorliegen. So. lange noch Heilungsvorgänge und demnach Heilungsmöglich. keiten vorhanden sind, spricht man von verzögerter Kno. chenheilung oder Konsolidation. Bei ihr ist eine Selbstheilung möglich, die Pseudarthrose kann nur operativ geheilt werden. So klar und bestimmt diese Richtlinien klingen, so schwierig oder gar unmöglich ist im Einzelfall die klinische Entscheidung, wo die verzögerte Knochenheilung aufhört und die Pseudarthrose anfängt; die Entscheidung ist ganz ins Belieben des einzelnen Chirurgen gestellt.

Unter den guten Erfolgen der operativen Pseudarthrosenbehandlung [Verbolzung oder Verzahnung der Bruchenden (Brun, Ringel, Francke), Vereinigung mit Laneschen oder anderen Platten (Geiges u.a.), seitliche Schienung ( $\mathrm{Guleke)}$ oder zentrale Bolzung (L ex e r) mit autoplastisch verpflanztem Knochen usw.] ist die' Behandlungsrichtung, auch für dic Grenzfälle, immer mehr eine operative geworden. Ganz abgesehen davon, daß die Operationen leider nicht regelmäßig zum erwünschten Ziele führen, zuweilen sogar vielleicht die Heilung eher erschweren, werden bei der operationsgeneigten Art der Indikationsstellung viele unnötige Operationen vorge nommen.

Wir halten es nach unseren Erfahrungen für notwendig, über der Neigung zur operativen Behandlung nicht zu vergessen, daß auch konservative Maßnahmen viele? „Pseudarthrosen“", 
die in Wirklichkeit nur lange verzögerte Konsolidationen sind, zur Heilung bringen können. Den Anlaß zur Mitteilung unserer Erfahrungen gibt folgende Beobachtung:

Am 12. XI. 1920 trat ein 36jähr. Mann mit ciner „Pseudarthrose" in der Mitte des 1. Oberarms in unsere Behandlung. Er hatte den Arm am 24. III. 1920 gebrochen, wurde in einem Krankenhaus mit Schienen- und Streckverbänden behandelt; da der Bruch nicht fest wurde, wurde er am 6. V. mit einem zentralen Elfenbeinstift ge. bolzt. Da wieder keine Festigkeit eintrat, wurde am 19. VII. als seitliche Schiene ein autoplastisch verpflanzter Tibiaspan angelegt. Wieder blieb die Festigkeit aus. $\mathrm{Mm}$ 12. XI. bestand an der Bruchstelle völlig freie Beweglichkeit; Röntgenbild: Die Bruchenden berühren sich in der einen Knochenhälfte bis auf einen schmalen Spalt; in der anderen Knochenhälfte sind sie durch eine etwa $1 / 2 \mathrm{~cm}$ breite Lücke getrennt. Der Bolzen sitzt im oberen Knochenende, seitliche Schiene nicht mehr erkennbar. Wir legten am 18. XI. einen einfachen Abduktionsgipsverband an, mit dem der Mann nach Hause reiste. Nach 6 Wochen (2. I. 1921) kam er wicder; die obere Hälfte des Armgipses wurde deckelförmig abgenommen, der Bruch war beinahe geheilt. Der Arm wurde jetzt, in der Hülse liegend, zu Hause massiert und bewegt. Nach weiteren 6 Wochen (8. II.) war der Bruch festgeheilt. Dieser Fall, der von den meisten wohl als .:Pseudarthrose" bezeichnet werden dürfte, ist demnach ohne Ope ration in 12 Wochen fest geheilt.

In gleicher Weise sind wir seit 1917 bei allen ähnlich verzögerten Knochenheilungen vorgegangen, die in unsere Behandlung kamen. Betroffen waren außer dem oben genannten Oberarm $13 \mathrm{mal}$ der Oberarm, $3 \mathrm{mal}$ der Ober-und $2 \mathrm{mal}$ der Unterschenkel. (Die Unterarmpseudarthrose bildet ein Gebiet für sich und scheidet, ebenso wie der Defektbruch des Schienbeins, wegen der Besonderheit der Verletzung eines zwciknochigen Gliedes, bei dieser Betrachtung aus, obwohl das hier gesagte cum grano salis auch für sic gilt.) Die geringen Verzögerungen der knöchernen Heilung um einige Wochen, die man nicht zu selten sicht, und die allgemein durch Gehgipse, Röntgenreizdosen usw. leicht beseitigt werden, sind natürlich nicht mitgerechnet. Es handelt sich vielmehr bei diesen i 8 Fällen um derartig verzögerte Heilungen, daß sie der gewöhnliche Sprachgebrauch meist als Pseud. arthrosen bezeichnen würde. Von diesen sind die 2 Unterschenkel, 2 Oberschenkel und 7 Oberarme 
o.hne operativen Eingriff, nur durch einfache Verbandbehandlung fest geworden.

Die Unterschenkelpse udarthrosen erhielten einen gut anliegenden Gehgipsverband, zuerst von den Zehen bis unter das Kniegelenk mit Gehbügel, dann nur eine einfache Gipshülse von den Knöcheln bis zum Knie: Sie waren in 4 Monaten festgeheilt.

Die 2 geheilten Oberschenkelpseudarthrosen heilten im Beckengehgipsverband in etwa $4 \frac{1}{2}$ Monaten völlig fest; der dritte, nicht fest gewordene Oberschenkel mußte mehrmals operiert werden.

Die 7 geheilten Oberarme wurden nur mit gutsitzendem Abduktionsgipsverband behandelt. Bei den 6 durch diese Verbandbehandlung nicht knöchern geheilten blieb die Heilung 4 mal deshalb aus, weil zwischen den beiden Knochenenden eine Lücke bestand, die sich durch einfaches Aneinanderpressen im Verband nicht beseitigen ließ und so ein Festwerden ohne Operation unmöglich machte. Der fünfte Oberarm wurde im Verband nicht fest, weil beide Knochenenden schon bindegewebig vernarbt waren (Operationsbefund). Von diesen 5 Oberarmen sind 4 nach einfacher operativer Verbolzung mit anschließendem Abduktionsverband rasch knöchern geheilt; der fünfte bekam kurz nach der Operation eine schwene Grippe, weshalb der Gipsverband nach kurzer Zeit abgenommen werden mußte, so daß der Knochen nicht heilen konnte. Der sechste Oberarm wurde auch nach mehrfachen Operationen der verschiedensten Art nicht fest.

Der Abduktionsgipsverband wird bei Schmerzen oder Gelenkkontrakturen in Plexusanästhesie angelegt, meist im Sitzen, bei schwachen Kranken im Liegen unter Verwendung einer langen, flachen Eisenstange, auf die der Kranke mit der Wirbelsäule der Länge nach gelegt wird, die aber nur am Kopf- und Beckenende unterstützt ist, so daß der Kranke völlig frei liegt; die Stange wird herausgezogen, sobald der Gips fest ist. Über den Wunden Fenster ! Über den 'Teilen des Gliedes, die nicht völlig festliegen müssen, wird die obere Hälfte des Verbandes in Form eines oberen Deckels aus. geschnitten. Den gleichen Verband wenden wir auch nach den Pseudarthrosen-Operationen an und halten ihn für deren Erfolg, cbenso wie Francke, für recht wichtig.

Einige Beispiele mögen erläutern, welche anscheinend aussichtslosen Fälle von verzögerter Knochenheilung so heilten : 
Fa11 I: R. $23 \mathrm{~J}$. I6. IX. I9I6 Schußbruch 1. UnterschenkelHitte. 19. V. 1917 Aufnahmebefund: völlig freie Beweglichkeit einer Unterschenkel-Pseudarthrose in der Mitte. 29. V. Fibrin-Injektion und Gehgips. Io. VIII. Fraktur fast fest. Gipshülse. 28. IX. Fraktur weiter fest. Neue Gipshülse. 6. XI. Knorhen fest, neue Gipshülse. 1. I. I9 8 Fraktur völlig fest.

SchluB: Eine $8^{1 / 2}$ Monate alte Unterschenkel-Pseudarthrose ist durch eine Gipsbehandlung von $4-5$ monatlicher Dauer völlig fest geworden. Auf die Fibrininjektion, die nicht in den Bruchspalt gelangt, fällt sicher kein Anteil am Erfolg.

Fall 2: Sch., 23 J., Unterschenkel-Schußbruch 23. IV. 1917, Mai 1917 auswärts Operationsversuch: Drahtnaht mit nachträglicher Extension. 28. XII. Aufnahmebefund: Freic Beweglichkeit einer Lnterschenkel-Pseudarthrose in der Mitte. I4. I. I918 Gipshülse. 20. I. erste Gehversuche. II. VI. Knochen beinahe, 20. VII. völlig fest.

SchluB : 8 Monate alte Unterschenkel-Pseudarthrose durch Gchgipsverband in $;$ Monaten fest geheilt.

Fall 3: H., 37 J., 9. XI. I9I6 Schußbruch r. Oberarm-Mitte. 18. XII. 1917 Aufnahmebefund: Völlig frei bewegliche Oberarm-Pseudarthrose in der Mitte. 12. XII. Abduktionsgips. 24. III. Oberarm fest geheilt. Gipsmanschette für einige Monate als Schutz.

Schluß: is Monate alte Oberarm-Pseudarthrose wird durch 3 Monate lange Abduktionsgipsbehandlung völlig fest.

Fall 4: C., 33 J. 2. VI. I917 Schußbruch r. Oberarm, handbreit unter dem Schultergelenk. 2I. VI. I9I8 Aufnahmebefund: fast völlig frei bewegliche Pseudarthrose. 26. VI. Abduktionsgips in $60 \mathrm{Grad}$ Abduktion. 30. IX. Knochen fest geheilt, Gips ab.

SchluB: Über 12 Monate alte Oberarm-Pseudarthrose heilte im Laufe cines Vierteljahres unter Abduktionsgipsbehandlung.

Diese Erfahrungen beweisen, da $B$ man mit einer derartigen konservativen Behandlung auch bei recht lange währendem Ausbleiben der knöchernen Heilung noch gute Erfolge haben kann. Der innere Grund dafür ist der, daß man die gute Knochenvereinigung und Ruhigstellung, denen die neueren Formen der Pseudarthrosenoperationen, wie Verbolzung, Verkeilung usw. mit ihre guten Erfolge verdanken, a u ch mit geeigneten Verbänden crzielen kann.

Unsere Behandlung der verzögerten Knochenheilung oder Pseudarthrose wird daher von folgenden Grundsätzen geleitet:

Bei Ober - und Unterschenkelbrüchen wird die sonst regelmäßig angewandte Streckverbandbehandlung abgebrochen, sobald die rechtzeitige knöcherne Heilung ausbleibt. An 
ihre Stelle tritt der fixierende Gipsverband, der neben der Ruhigstellung auch die Belastung gestattet. In derselben Weise werden Beinbrüche behandelt, die im Zustand der verzögerten Heilung bzw. als scheinbare Pseudarthrosen zu uns kommen. Vicle werden so' noch fest werden.

Am $\mathrm{O}$ be ra r $\mathrm{m}$ ist es bei verzögerter Knochenheilung und dadurch drohender Pseudarthrose die erste Pflicht, nicht bloß auf die Extensionsbehandlung, die bei der Oberarmfraktur im allgemeinen überhaupt nur sehr milde angewandt $\mathrm{zu}$ werden braucht, zu verzichten, sondern auch die Dauerextension des Bruches durch den schweren, fortwährend an der Bruchstelle hängenden Unterarm auszuschalten und einen gutsitzenden Abduktionsgipsverband anzulegen; Schienenabduktionsverbände oder Abduktionsapparate genügen keinesfalls für die sichere Ruhigstellung. Um die schädlichen Folgen der dauernden Feststellung gerade am Arm zu vermeiden, wird man so bald als möglich den oberen Teil des Gipsverbandes deckelförmig abschneiden. Auch nach Pseudarthrosenoperationen ist eine gute Gipsbehandlung, am Oberarm eine Abduktionsgipsbehandlung von wesentlicher, vielleicht ausschlaggebender Bedeutung.

Neben der Verbandbehandlung werden natürlich auch Röntgenbestrahlung, entsprechende Einspritzungen und alle anderen kleineren konservativen Mittel angewandt, wobei wir besonders von der R ö nt genbestrahlung manch Gutes gesehen zu haben glauben.

Behandelt man alle verzögerten Knochenheilungen grundsätzlich mit derartigen Verbänden, so sind nach 6 Wochen manche schon fest geheilt - uns heilte cin Oberarm in 5 Wochen -; jedenfalls erkennt man nach 6 bis 8 Wochen die F älle, welche mit einfacher Verbandbehandlung fest werden, die demnach nur verzögerte Heilungen sind, und die, welche so nicht heilen können und operiert werden müssen, die demnach wirkliche Pseudarthrosen sind. Die auBer bei Defektbrüchen sehr schwicrige oder unmög. liche Entscheidung, ob es sich nur um eine verzögerte Heilung oder schon um eine Pseudarthrose. handelt, wird·demnach durch eine etwa 2 monatliche Beobachtung sicher getroffen. Durch diese kurze, nach unserer Ansicht unbedingt nötige Bcobachtung wird nicht $z u$ viel Zeit verloren, auch wenn nachträglich eine operative Behandlung der Pseudarthrose nötig wird. 